\title{
RADIATION-INDUCED POLYGENIC MUTATION IN ARABIDOPSIS THALIANA
}

\section{ANALYSIS OF LINES SELECTED FOR NUMBERS OF SEEDS PER POD}

\author{
C. W. LAWRENCE* \\ Wantage Research Laboratory (AERE), Wantage, Berks., U.K.
}

Received 14.v.74

\begin{abstract}
SUMMARY
The genetic variation between inbred lines, previously selected for high or low numbers of seed per pod in families arising from plants irradiated with 100 , 200 , or 300 krads of ${ }^{60} \mathrm{Co}$ gamma rays, was analysed by constructing a $13 \times 13$ set of diallel crosses. The analysis showed that the induced mutations were exclusively recessive and suggested the presence of non-allelic interactions. No evidence was found for mutations increasing the number of seeds per pod. In this respect, the induced mutations affecting the number of seeds per pod contrast with those influencing flowering time, since, with these, approximately equal numbers act in the early and late directions (Lawrence, 1968a). Similarly, flowering time mutations can be either dominant or recessive, dominance is generally incomplete, and there is no evidence for non-allelic interaction (Lawrence, 1968b).

Two out of six low selection lines included in the $13 \times 13$ diallel, and one high line, appeared to be homozygous for interchanges or similar chromosome aberrations and these may be responsible for the marked lack of linearity in the relation between selection response and dose. Correlated changes in the unselected character flowering time were negligible in lines selected for number of seeds per pod and vice versa, though one extremely late and infertile line was obtained.
\end{abstract}

\section{INTRODUGTION}

Previous papers in this series have examined the selection (Lawrence, 1968a) and analysis (Lawrence, 1968b, 1975a) of radiation-induced quantitative variation in flowering time, and the selection (Lawrence, 1975b) of variation in the number of seeds per pod in the crucifer Arabidopsis thaliana (Heyn.). The present report is concerned with the genetic analysis, by means of diallel crosses, of the lines selected for number of seeds per pod and summarises the conclusions from the series of experiments.

The overall objective of this series was to compare results from these two characters, one probably a major and the other a minor component of fitness, with respect to the genetic properties of the induced micromutations and also to the relative rates of induction of such mutations in the " high" and "low" directions. Such information should be useful both from a theoretical point of view, revealing the dependence of the genetic properties of induced mutations on the "genetic architecture" (Mather, 1960) of the character studied and, from a practical view point, aiding the evaluation of the potential use of induced micro-mutations in programmes of crop plant improvement.

* Present address : Department of Radiation Biology and Biophysics, School of Medicine and Dentistry, The University of Rochester, Rochester, New York 14642, U.S.A. 


\section{Material and methods}

The origin of the inbred lines used as parents in the $13 \times 13$ set of diallel crosses can be obtained from the previous paper (Lawrence, 1975b). The lines include two selected for high, and two for low numbers of seeds per pod from each of the three dose groups (100,200, and $300 \mathrm{krads})$ and a high selection from the control. All crosses were carried out under conditions of rigorous isolation in a controlled environment room. Seed from each cross were taken from at least three pods to avoid maternal effects. Despite repeated attempts at crossing, 100Ll, a low selection line from the $100 \mathrm{krad}$ group, proved to be almost entirely sterile as male parent while 300L2, a low selection line from the $300 \mathrm{krad}$ group, was almost entirely sterile as female parent. Reciprocal crosses were fertile in both cases.

The 146 selfs and crosses comprising the remainder of the $13 \times 13$ set of diallel crosses were grown in soil, according to methods described previously (Lawrence, 1968a), in a single trial composed of eight blocks with five plants per plot. The average number of seeds per pod was estimated from the first five pods of all surviving plants, harvested just before maturity and stored in 70 per cent ethanol for at least one day before examination with the aid of a binocular microscope. For further details, see Lawrence $(1975 b)$. The plants were also scored for flowering time, that is the number of days between sowing and the opening of the first flower.

In addition, the number of seeds per pod was recorded in plants from a $7 \times 7$ set of diallel crosses, the inbred parents of which had been selected for either early or late flowering. The flowering time data, details concerning experimental design and derivation of the parental lines have already been published (Lawrence, 1968b).

\section{RESULTS}

\section{(i) Fertility in the $13 \times 13$ diallel}

The average number of seeds per pod in each of the 13 homozygous lines and 133 hybrids, comprising all of the crosses that could be made, is given in table 1 . As expected from previous results (Lawrence, 1975b), it is evident from this table that there is considerable variation among the families, particularly in the direction of low fertility. These data provide no evidence for the induction of mutations which increase the number of seeds per pod, however, and the highest entry in table 1 is that for the homozygous control line $0 \mathrm{H} 1$. Similarly, all of the array averages, pooled over reciprocal crosses, are lower than the average of the control array, with the exception of the $200 \mathrm{H} 2$ array which is only fractionally and insignificantly higher.

Before the significance of this variation can be assessed, by means of the analysis of variance for diallel tables proposed by Yates (1947) and Hayman (1954), two problems must be examined. First, inspection of table 1 shows that all of the hybrid progeny of three lines $(200 \mathrm{~L} 4,300 \mathrm{H} 2,300 \mathrm{~L} 4)$ consistently show "negative heterosis", that is the number of seeds per pod in each of the hybrids is considerably lower than that of the lower parent. Although it is possible that this result originates in the induction of point mutations which exhibit strong gene-gene interactions, the most likely 


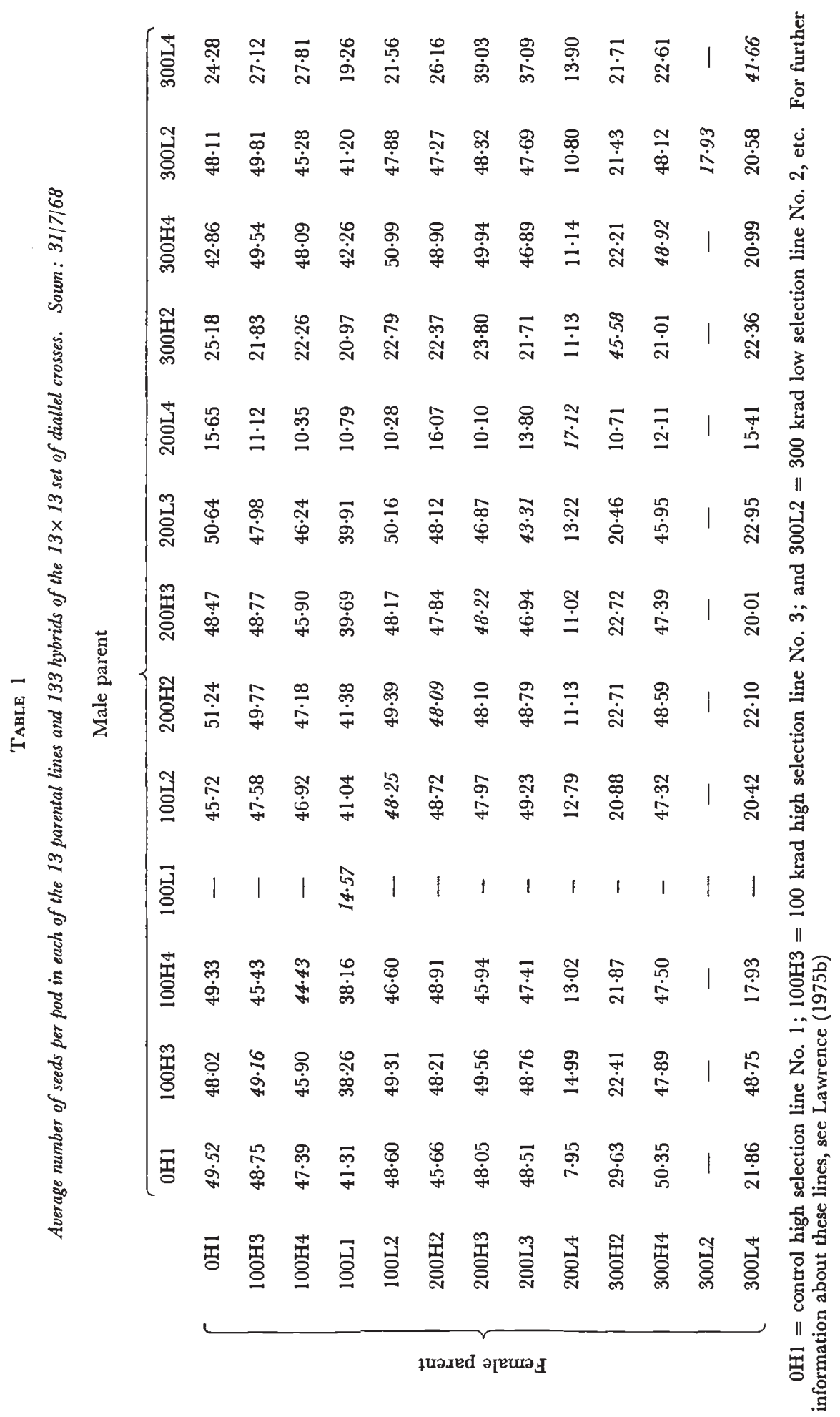


explanation is that the three parental lines are homozygous for interchanges or similar chromosome aberrations. Since aberrations of these kinds will, in the heterozygous condition, affect fertility but probably not flowering time, and since the object of the experiment is to study genetic variation of a more normal variety, these lines and their hybrid progeny were omitted from the data that were analysed.

The second problem with the data summarised in table 1 is how best to make allowance for the results missing from the $100 \mathrm{Ll}$ male and $300 \mathrm{~L} 2$ female arrays. Since the missing data pertain solely to the consistent $(c)$ and inconsistent $(d)$ variation between reciprocal crosses, the significance of these items was first examined in the $8 \times 8$ diallel table which results from excluding observations from all of the progeny of $100 \mathrm{~L} 1$ and $300 \mathrm{~L} 2$ (as well as the progeny of 200L4, 300H2 and 300L4). Since neither of

TABLE 2

Analysis of variance in number of seeds per pod in the $10 \times 10$ diallel

\begin{tabular}{|c|c|c|c|}
\hline Item & d.f. & M.S. & V.R. \\
\hline$a$ & 9 & $1510 \cdot 68$ & $50 \cdot 87 * * *$ \\
\hline$b$ & 45 & $196 \cdot 37$ & $10 \cdot 96 * * *$ \\
\hline$b 1$ & 1 & $1955 \cdot 24$ & $315 \cdot 35 * * *$ \\
\hline$b 2$ & 9 & 695.93 & $28 \cdot 39 * * *$ \\
\hline$b 3$ & 35 & $17 \cdot 65$ & 1.07 n.s. \\
\hline$c$ & 7 & $9 \cdot 62$ & $<1$ n.s. \\
\hline$d$ & 21 & $23 \cdot 83$ & 1.58 n.s. \\
\hline$a \times$ blocks & 63 & $29 \cdot 69$ & - \\
\hline$b \times$ blocks & 315 & $17 \cdot 92$ & - \\
\hline$b 1 \times$ blocks & 7 & $6 \cdot 20$ & - \\
\hline b2 2 blocks & 63 & $24 \cdot 51$ & - \\
\hline$b 3 \times$ blocks & 245 & $16 \cdot 56$ & - \\
\hline$c \times$ blocks & 49 & $21 \cdot 75$ & - \\
\hline$d \times$ blocks & 139 & $15 \cdot 11$ & - \\
\hline Blocks & 7 & $452 \cdot 42$ & $23.97 * * *$ \\
\hline Total & 655 & - & \\
\hline
\end{tabular}

these items was significant, it appeared permissible to fill in the missing data, block by block, using results from the reciprocal cross and dropping an appropriate number of degrees of freedom in the analysis of the $10 \times 10$ table.

The result of this analysis is given in table 2. It shows the existence of highly significant additive $(a)$ and non-additive $(b)$ genetic variation between the 10 parental lines. The non-additive variation was due principally to a consistent deviation of hybrids from their mid-parent value $\left(b_{1}\right)$, though there was also variation between lines in non-additive effects $\left(b_{2}\right)$. As noted before, there was no evidence for either consistent $(c)$ or inconsistent $(d)$ variation between reciprocal crosses. Since the various interaction mean squares (main effects $\times$ blocks) were significantly heterogenous, each main effect was tested against the appropriate estimate or error variation.

Closer examination of the non-additive variation, using the variances $(V r)$ and covariances $(W r)$ of arrays according to the method of Jinks 
(1954), shows (fig. 1) that nearly all of this item can be ascribed to the lines $100 \mathrm{Ll}$ and $300 \mathrm{~L} 2$, which contain mutations that are recessive compared to those carried by the control and the remaining lines. Since there is a high and significant negative correlation between $(V r+W r)$ and the array mean $(r=-0.752, \mathbf{P}=0.02-0 \cdot 01)$ it is clear that dominance acts exclusively in the direction of high fertility. The regression of $W r$ on $V r$ is highly significant $(P<0.001)$, with a slope which deviates significantly from the value of one expected in the absence of epistasis $(b=+0 \cdot 876 \pm 0 \cdot 017$,

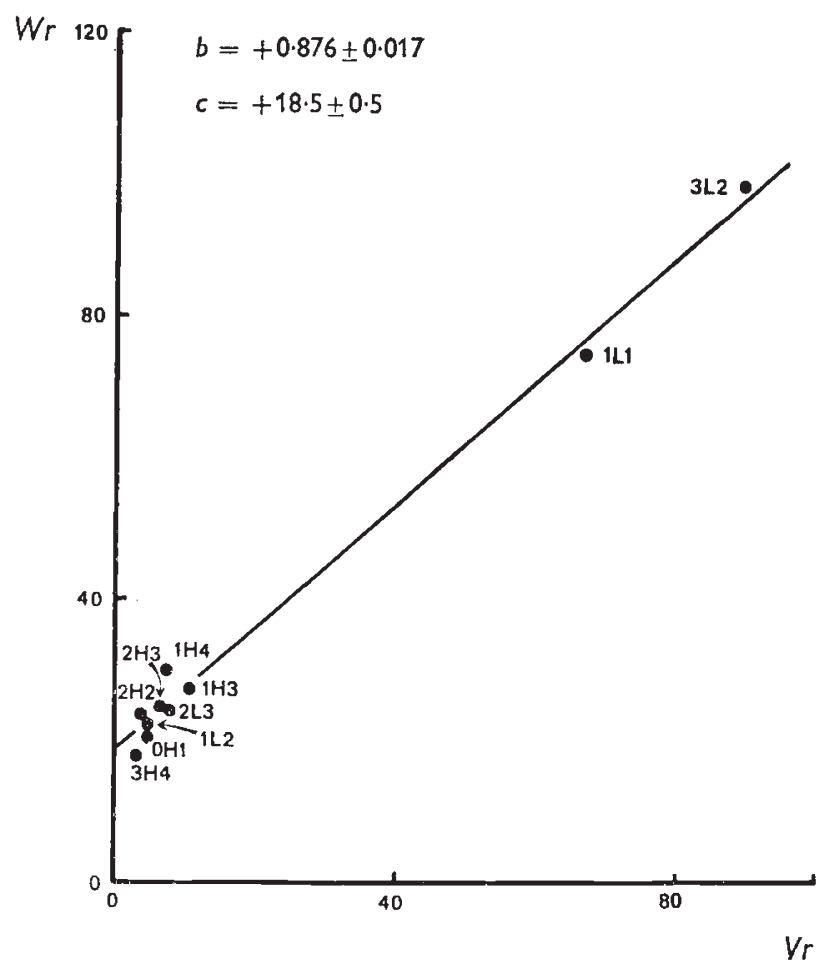

FIG. 1.- $W r / V r$ graphs for number of seeds per pod in the $10 \times 10$ diallel table. For further details concerning this method of analysis, see Jinks (1954).

deviation from $+1 \cdot 000, P<0 \cdot 001)$. Similarly, the intercept of the regression line on the $W r$ axis, numerically equal to $\frac{1}{4}\left(\mathrm{D}-\mathrm{H}_{1}\right)$, is significantly different from zero $(c=+18.5 \pm 0 \cdot 5, \mathrm{P}<0 \cdot 001)$. Together, these items may indicate the existence of epistatic gene interactions among the induced mutations, and in the presence of such presumed interaction it is difficult to say whether dominance is complete or incomplete. All variances and covariances used in this analysis were corrected appropriately for error variation. To summarise, analysis of the $10 \times 10$ set of diallel crosses provides no evidence for the existence of mutations which increase the number of seeds per pod; on the contrary, all induced mutations decrease fertility. Such mutations are always recessive and may exhibit epistatic interactions with one another or with control alleles. 


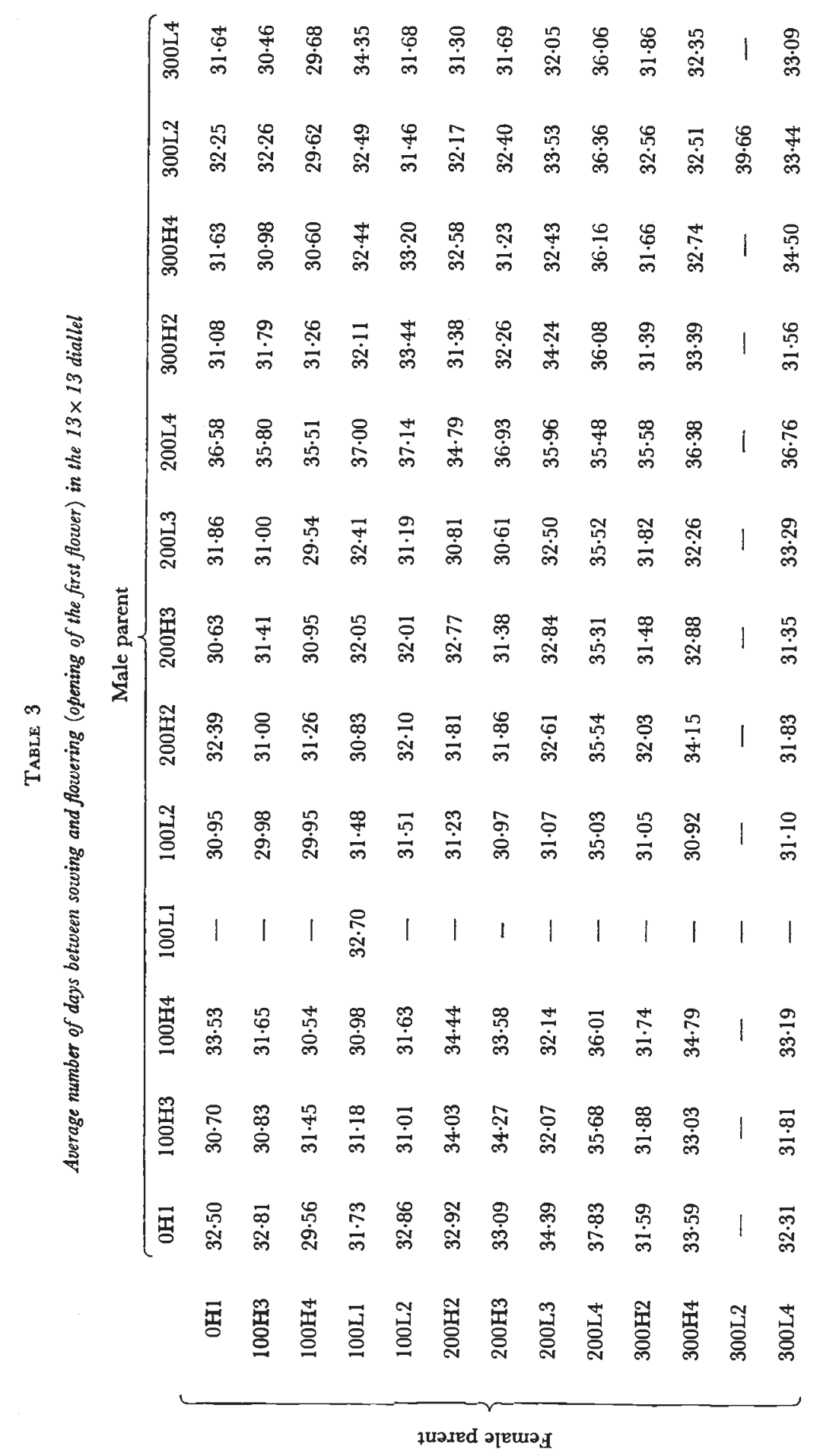




\section{(ii) Flowering time in the $13 \times 13$ diallel}

Apart from the number of seeds per pod, flowering time was also recorded in the $13 \times 13$ set of diallel crosses and these data are given in table 3 . In general, there is rather little variation with respect to this unselected character, with some lines flowering earlier and others later than the control, though some of the low lines flower appreciably later than normal. There is no evidence that the chromosome interchanges, presumed to be present in the $300 \mathrm{H} 2$ and $300 \mathrm{~L} 4$ lines, have much influence on flowering time, either when in the homozygous or the heterozygous condition. The 200L4 line, and all its hybrid progeny, flower rather later than normal, however, though this could be due to mutations independent of the presumed interchange.

Analysis of the $13 \times 13$ diallel table (table 4 ) in the manner used previously for fertility shows the presence of significant additive $(a)$ and non-additive

TABLE 4

Analysis of variance in flowering time in the $13 \times 13$ diallel

\begin{tabular}{|c|c|c|c|}
\hline Item & d.f. & M.S. & V.R. \\
\hline$a$ & 12 & $252 \cdot 50$ & $6 \cdot 49 * *$ \\
\hline$b$ & 78 & $13 \cdot 01$ & $1 \cdot 82 *$ \\
\hline$b 1$ & 1 & 1.67 & $<1$ n.s. \\
\hline$b 2$ & 12 & 43.03 & $6 \cdot 02 * * *$ \\
\hline$b 3$ & 65 & $7 \cdot 64$ & 1.09 n.s. \\
\hline$c$ & 10 & 38.93 & $14 \cdot 77 * * *$ \\
\hline$d$ & 45 & $7 \cdot 15$ & $2 \cdot 71 * * *$ \\
\hline Blocks & 7 & $169 \cdot 25$ & $64 \cdot 11 * * *$ \\
\hline Error & 1017 & $2 \cdot 64$ & - \\
\hline Total & 1162 & - & - \\
\hline
\end{tabular}

(b) variation. Unlike the situation with fertility, there is no evidence for a consistent deviation of hybrids from the midparent value $\left(b_{1}\right)$ though individual lines have characteristic non-additive effects $\left(b_{2}\right)$. As found before (Lawrence, 1968b) for flowering time, there is both consistent $(c)$ and inconsistent $(d)$ variation between reciprocal crosses. The regression of $W r$ and $V r$ is not significant. It would appear, therefore, that a more or less random selection of mutations affecting flowering time were fixed in the parental lines, though with some tendency for the low selection lines to contain mutations which retard flowering. Such mutations do not appear to exhibit dominance exclusively in one direction.

These results suggest either that the set of mutations affecting flowering time were, at least in part, different from those affecting fertility or that there is no specific pleiotropic relation between flowering and number of seeds per pod. This was examined further by means of correlations between these two characters in the $10 \times 10$ set of diallel crosses, pooling results from reciprocal crosses. Overall there is a highly significant negative correlation between number of seeds per pod and number of days from sowing to flowering $\left(r=-0.462, \mathrm{P}_{(53)}<0.001\right)$, but inspection of tables 1 and 3 suggests that this may be due entirely to one line, 300L2, which 
exhibits both very low fertility and late flowering. Removal of this one line removes all evidence of the correlation $(r=+0.013)$. Similarly, the correlation is not significant among the high lines and their hybrids $(\mathrm{P}=$ $+0.387)$ or among the low lines and their hybrids $(r=-0 \cdot 176)$ if 300L2 (but not its hybrid progeny) is excluded. Evidently mutations affecting flowering time and fertility are either substantially independent of one another or if not, affect the two characters in varying ways. Exceptionally, mutations or combinations of mutations may be induced which so reduce vitality and growth that fertility is reduced and flowering delayed simultaneously as in 300L2.

\section{(iii) The $7 \times 7$ diallel}

These data (table 5) provide the reverse of those above, since fertility is now the unselected, and flowering time the selected, character. Two of the lines, $R_{1} E$ and $R_{3} L_{2}$, would appear to contain interchanges since fertility in all their hybrids is substantially lower than in the lower parent. As before, these were excluded for the purpose of analysis. Results of the analysis of the remaining $5 \times 5$ set of diallel crosses are given in table 6 . They show evidence of highly significant additive variation (a) between the lines but, surprisingly, no evidence of significant non-additive variation. No correlation is evident between flowering time and number of seeds per pod,

TABLE 5

Average number of seeds per pod in the $7 \times 7$ diallel. Sown: $4 / 7 / 67$

$\begin{array}{lccccccc} & \text { Control } & \mathrm{S}_{3} \mathrm{E} & \mathrm{S}_{2} \mathrm{~L}_{1} & \mathrm{~A}_{3} \mathrm{E} & \mathrm{A}_{1} \mathrm{~L}_{1} & \mathrm{R}_{\mathbf{1}} \mathrm{E} & \mathrm{R}_{3} \mathrm{~L}_{2} \\ \text { Control } & 50 \cdot 40 & 47 \cdot 78 & 48 \cdot 88 & 48 \cdot 55 & 44 \cdot 52 & 35 \cdot 56 & 23 \cdot 60 \\ \mathrm{~S}_{3} \mathrm{E} & 47 \cdot 34 & 47 \cdot 28 & 48 \cdot 56 & 47 \cdot 41 & 42 \cdot 18 & 32 \cdot 96 & 22 \cdot 48 \\ \mathrm{~S}_{2} \mathrm{~L}_{1} & 48 \cdot 99 & 47 \cdot 62 & 49 \cdot 09 & 48 \cdot 12 & 44 \cdot 94 & 35 \cdot 80 & 22 \cdot 78 \\ \mathrm{~A}_{3} \mathrm{E} & 48 \cdot 77 & 46 \cdot 55 & 49 \cdot 21 & 47 \cdot 68 & 43 \cdot 84 & 34 \cdot 18 & 23 \cdot 81 \\ \mathrm{~A}_{1} \mathrm{~L}_{1} & 45 \cdot 55 & 43 \cdot 20 & 44 \cdot 54 & 41 \cdot 96 & 41 \cdot 29 & 30 \cdot 75 & 22 \cdot 37 \\ \mathrm{R}_{1} \mathrm{E} & 35 \cdot 39 & 33 \cdot 95 & 35 \cdot 53 & 35 \cdot 69 & 31 \cdot 81 & 46 \cdot 35 & 19 \cdot 12 \\ \mathrm{R}_{3} \mathrm{~L}_{2} & 23 \cdot 74 & 22 \cdot 06 & 22 \cdot 39 & 20 \cdot 38 & 20 \cdot 22 & 17 \cdot 74 & 38 \cdot 90\end{array}$

TABLE 6

Analysis of variance in number of seeds per pod in the $5 \times 5$ diallel

\begin{tabular}{|c|c|c|c|}
\hline Item & d.f. & M.S. & V.R. \\
\hline$a$ & 4 & $296 \cdot 66$ & $35 \cdot 99 * * *$ \\
\hline$b$ & 10 & $5 \cdot 92$ & $<1$ n.s. \\
\hline$b 1$ & 1 & $16 \cdot 72$ & 2.03 n.s. \\
\hline$b 2$ & 5 & $2 \cdot 85$ & $<1$ \\
\hline$b 3$ & 4 & $4 \cdot 82$ & $<1$ \\
\hline$c$ & 4 & 1.75 & $<1$ \\
\hline$d$ & 6 & $4 \cdot 72$ & $<1$ \\
\hline Blocks & 7 & $148 \cdot 53$ & $18 \cdot 02 * * *$ \\
\hline Error & 168 & $8 \cdot 24$ & - \\
\hline Total & 199 & - & - \\
\hline
\end{tabular}


either in all of the data $(r=-0.063)$ or in the high or low lines and their hybrids take separately $(r=+0 \cdot 112, r=+0.072)$. The flowering time data can be seen in an earlier report (Lawrence, 1968b, table 2).

\section{Discussion}

Apart from establishing that the mutations affecting flowering time and fertility were largely independent of one another, the analysis of lines selected for high and low numbers of seed per pod showed that all induced mutations influencing this character were recessive; there were no dominant alleles in the irradiated lines. In addition, the analysis suggested that the mutations exhibited non-allelic interactions with control alleles or among themselves, and in confirmation of earlier results provided no evidence for the existence of mutations that increase the number of seed per pod.

The mutations affecting the number of seeds per pod therefore contrast with those influencing flowering time in all respects. Flowering time mutations are about as likely to increase as decrease flowering time, dominance is usually incomplete and often ambidirectional and there is no evidence for the presence of non-allelic interactions (Lawrence, 1968b). Such differences can hardly stem from minor variations in experimental design. More probably they reflect fundamental and characteristic differences in the gene systems controlling flowering and fertility, particularly specific differences in gene action and interaction. It has been argued that these properties, which have been termed the "genetic architecture" of the character (Mather, 1960), arise as a result of natural selection (Fisher, 1930; Mather, 1953; Jinks, 1955). Natural selection presumably favours some intermediate range of flowering times, that is the character is exposed to stabilising selection. Very early flowering plants tend to produce very small leaf rosettes and hence small amounts of seed while very late plants may become overgrown or even fail to flower at all. Stabilising selection is thought to favour weak ambidirectional dominance and the absence of non-allelic interaction (Breese and Mather, 1957), and this is of course exactly what is seen with regard to flowering time in the present experiments.

The number of seeds per pod might be expected, on the other hand, to be exposed largely to directional selection since, within limits, natural selection will favour a high number of seeds. Directional selection is thought to result in strong directional dominance and the presence of non-allelic interactions (Breese and Mather, 1960), and again this is largely what is found for fertility in the present experiments. Evidence supporting this hypothesis, drawn from their own experiments and those of others, has been summarised by Kearsey and Kojima (1967).

The series of experiments with Arabidopsis would therefore appear to indicate that virtually all of the induced mutations decrease fitness and exhibit properties appropriate for such " negative" alleles in the various gene systems examined. Results compatible with this view have also been obtained by Dalebroux and Kojima (1967). Whether this is merely because the induced mutations were deletions or other gross alterations of the genome is not known, though by analogy with micro-organisms it seems likely that at least some and perhaps most of the mutations arose from 
alterations of only a few base pairs. If so, the characteristic properties of the mutations presumably arose from their interaction with other loci in the manner suggested by Fisher (1930), and it can be argued that gene systems are, in one sense, preadapted to cope with newly arising mutations. Similar non-random properties may well be found with spontaneous mutations also.

It should be emphasised that abnormal plants were carefully screened from the experiments, and the results pertain only to plants judged to be " normal" by visual examination. The experiments therefore provide no support for the suggestion that micro-mutations are more likely to improve fitness than macro-mutations (Gregory, 1965), though it is of course possible that a different result would have been found if mutations with even smaller effect had been studied. Such mutations are, however, unlikely to be selected in normal circumstances, and are unlikely to give a detectable response. From the point of view of an assessment of the potential value of induced micro-mutations for crop plant improvement, the experiments therefore suggest that greatest success will be obtained with characters, such as flowering time, that are not directly related to fitness. Improve ments with respect to traits, such as grain yield, that are more directly related to fitness would appear to be much less probable. A similar assessment has been made by others (e.g. Griffith and Johnson, 1962; Sakai and Suzuki, 1964), though by far the greater number of those concerned with this problem have arrived at a much more optimistic conclusion (Scossiroli, 1965; Brock, 1965; Gregory, 1968; Gaul et al., 1969; Gardner, 1969). In some instances, the importance of the relation between the character studied and fitness has not been appreciated, and success with minor components of fitness has led to the suggestion that yield will behave similarly. Others, notably Gregory (1968), however, have always insisted that increase in yield is the main criterion by which micro-mutation breeding is to be judged. In other instances the optimism stemmed from predicted gains in yield, based on data from early generations (Rawlings et al., 1958; Matsuo and Onozawa, 1961 ; Jalil and Yamaguchi, 1965), but such predictions are misleading and where tested the expected response to selection often failed to materialise (e.g. Papa et al., 1961). The best case for micro-mutation breeding comes from the extensive and detailed work of Gregory (1968) and Gaul et al. (1969) both of whom have demonstrated substantial improvements in yield following selection in irradiated populations. Both series of experiments also showed detectable, though smaller, responses in control populations, however, and, as pointed out previously (Lawrence, 1975b), where the starting material is genetically heterogeneous, irradiation may merely improve the utilisation of pre-existing mutations rather than provide new variation.

To summarise, it is concluded that estimates of the proportion of mutations increasing fitness, in an agricultural context, have been much inflated and that the true value is substantially smaller. In the present experiments, where genetic homogeneity of the control was clearly demonstrated, the proportion of positive mutations may have been somewhat underestimated due to chance fixation of negative alleles in the lines selected for high fertility. An occurrence of this kind is not unique to this experiment, of course, and will be found in all inbreeding species because negative mutations far outnumber positive ones. Such fixation can best be avoided by intercrossing the selected lines followed by further selection. Adoption of this 
procedure for inbreeding crop plants makes micro-mutation breeding much less attractive, however, though it would not necessarily be a disadvantage with crossbreeding species.

Acknowledgement.-I would like to thank Mrs M. Richold and Mr D. Cooke for their skilled assistance in this work. This paper was written when under contract with the U.S.A.E.C. at the University of Rochester Atomic Energy Project, and is assigned Report No. UR-3490-691.

\section{REFERENGES}

BREESE, E. L., AND MATHER, K. 1957. The organisation of polygenic activity within a chromosome in Drosophila. I. Hair characters. Heredity, 11, 373-395.

BREESE, E. L., AND MATHER, K. 1960. The organisation of polygenic activity within a chromosome in Drosophila. II. Viability. Heredity, 14, 375-399.

BROCK, R. D. 1965. Induced mutations affecting quantitative characters. The use of induced mutations in plant breeding. Rad. Bot., 5 (Suppl.), 451-464.

DALEBROUX, M. A., AND KoJIMA, K. I. 1967. An analysis of radiation-induced variation on body weight of Habrobracon juglandis. Genetics, 55, 315-328.

Fisher, R. A. 1930, 1958. The Genetical Theory of Natural Selection, 2nd ed. Dover, New York.

GARDNER, C. O. 1969. Genetic variation in irradiated and control populations of corn after ten cycles of mass selection for high grain yield. In Induced Mutations in Plants, pp. 469-477. I.A.E.A., Vienna.

GAUl, H. P. K., ULONSKA, E., ZUM WINKEL, C., AND BRAKER, G. 1969. Micro-mutations influencing yield in barley-studies over nine generations. In Induced Mutations in Plants, pp. 375-398. I.A.E.A., Vienna.

GREGORY, w. c. 1965. Mutation frequency, magnitude of change and the probability of improvement in adaptation. The use of induced mutations in plant breeding. Rad. Bot., 5 (Suppl.), 429-441.

GREGORY, w. C. 1968. Review Paper. A radiation breeding experiment with peanuts. Rad. Bot., 8, 81-147.

GRIFFITH, D. J., AND JOHNSON, T. C. 1962. The use of an irradiation technique in oat breeding. Rad. Bot., 2, 41-51.

HAYMAN, B. I. 1954. The analysis of variance of diallel tables. Biometrics, 10, 235-244.

JALIL, M. A., AND YAMAGUCHI, H. 1965. The variation of quantitative characters in the irradiated progenies of two rice varieties and their hybrids. Rad. Bot., 5, 187-196.

JiNks, J. L. 1954. The analysis of continuous variation in a diallel cross of Nicotiana rugtiea varieties. I. The analysis of $\mathrm{F}_{1}$ data. Genetics, 39, 767-788.

JINKs, J. L. 1955. A survey of the genetical basis of heterosis in a variety of diallel crosses. Heredity, 9, 223-238.

KEARSEY, M. J., AND KOJIMA, K. I. 1967. The genetic architecture of body weight and egg hatchability in Drosophila melanogaster. Genetics, 56, 23-37.

LAWRENCE, C. W. 1968a. Radiation-induced polygenic mutation in Arabidopsis thaliana. I. Selection for flowering time. Heredity, 23, 321-337.

LAWRENCE, C. W. 1968b. Radiation-induced polygenic mutation in Arabidopsis thaliana. II. Analysis of lines selected for flowering time. Heredity, 23, 573-589.

LAWRENCE, C. W. 1975a. Radiation-induced polygenic mutation in Arabidopsis thaliana. III. Effect of parental genotype and the limits to change. Heredity, 34, 189-201.

LAWRENCE, C. W. 1975b. Radiation-induced polygenic mutation in Arabidopsis thaliana. IV. Selection for number of seeds per pod. Heredity, 34, 203-212.

MATHER, K. 1953. Genetical control of stability in development. Heredity, 7, 297-336.

MATHER, K. 1960. Evolution in polygenic systems. Evolution e Genetica, Accad. Nazionale die lincei, 47, 131-152.

MATSUO, T., AND ONOZAWA, Y. 1961. Studies on mutation by radiation and chemicals (2). Japan F. Breeding, 11, 295-299.

PAPA, K. E., WILliaMs, J. H., AND HANWAY, D. G. 1961. Effectiveness of selection for quantitative characters in the third generation following irradiation of soybean seed with $\mathrm{X}$-rays and thermal neutrons. Crop Sci., 1, 87-90. 
RAWLiNGs, J. O., HANWAY, D. G., AND GARDNER, G. o. 1958. Variation in quantitative characters of soybeans after seed irradiation. Agron. Four., 50, 524-528.

SAKAI, K. I., AND sUzUKI, A. 1964. Induced mutation and pleiotropy of genes responsible for quantitative characters in rice. Rad. Bot., 4, 141-151.

SCOSSIROLI, R. E. 1965. Value of induced mutations for quantitative characters in plant breeding. The use of induced mutations in plant breeding. Rad. Bot., 5 (Suppl.), 443-450.

yATEs, F. 1947. Analysis of data from all possible reciprocal crosses between a set of parental lines. Heredity, 1, 287-301. 